\title{
Diel flight activity of wild-caught Anopheles farauti (s.s.) and An. hinesorum malaria mosquitoes from northern Queensland, Australia
}

Giles E. Duffield ${ }^{1 *}$, Dominic J. Acri ${ }^{1}$, Gary F. George ${ }^{1}$, Aaron D. Sheppard ${ }^{1}$, Nigel W. Beebe ${ }^{2,3}$, Scott A. Ritchie ${ }^{4}$ and Thomas R. Burkot ${ }^{4}$

\begin{abstract}
Background: Species in the Anopheles farauti complex are major malaria vectors in the Asia Pacific region. Anopheline mosquitoes exhibit circadian and diel rhythms in sugar- and blood-feeding (biting), flight activity, oviposition, and in some species, a short-lived dusk/early night associated swarming behaviour during which mating occurs. A behavioural study of wild-caught mosquitoes from Queensland, Australia was conducted to investigate the differences in diel rhythmic flight activity between two cryptic species in several reproductive states.

Results: The 24-hour flight activity of individual adult female mosquitoes under light:dark cycle conditions were monitored with a minute-to-minute time resolution using an infrared beam break method. Mosquitoes were analyzed for reproductive state (insemination and parity) and identified to species [An. farauti (s.s.) Laveran and An. hinesorum Schmidt] by PCR analysis. We compared daily total flight activity, timing of activity onset, the peak in early nocturnal activity, and patterns of activity during the scotophase (night). Species-specific differences between $A n$. farauti and An. hinesorum were observed. Compared to An. farauti, An. hinesorum had an earlier onset of dusk activity, an earlier peak in nocturnal activity, and a higher level of activity at the onset of darkness. Small differences between species were also observed in the pattern of the dusk/early-night bouts of activity. A second nocturnal peak in inseminated nulliparous An. hinesorum was also observed during the middle of the scotophase.

Conclusions: The behavioural differences between these two sympatric species of the An. farauti complex might contribute to subtle differences in habitat adaptation, the timing of host-seeking and/or sugar-feeding activity. This study provides baseline data for analysis of populations of mosquitoes from other geographical regions where these species are malaria vectors, such as in the Solomon Islands and Papua New Guinea. This is important as selective pressures due to long-term use of indoor residual spraying of insecticides and insecticide-treated bed nets are shifting the nocturnal profile of biting behaviour of these vectors to earlier in the night.
\end{abstract}

Keywords: Mosquitoes, Anopheles farauti, Anopheles hinesorum, Circadian clock, Diel rhythm, Locomotor activity, Malaria

\footnotetext{
* Correspondence: duffield.2@nd.edu

'Department of Biological Sciences and Eck Institute for Global Health, Galvin

Life Science Center, University of Notre Dame, Notre Dame, IN 46556, USA

Full list of author information is available at the end of the article
}

(c) The Author(s). 2019 Open Access This article is distributed under the terms of the Creative Commons Attribution 4.0 International License (http://creativecommons.org/licenses/by/4.0/), which permits unrestricted use, distribution, and reproduction in any medium, provided you give appropriate credit to the original author(s) and the source, provide a link to the Creative Commons license, and indicate if changes were made. The Creative Commons Public Domain Dedication waiver (http://creativecommons.org/publicdomain/zero/1.0/) applies to the data made available in this article, unless otherwise stated. 


\section{Background}

Mosquitoes of the Anopheles farauti complex (An. punctulatus group) are major vectors of human malaria in Indonesia, Papua New Guinea (PNG), the Solomon Islands, Vanuatu and, previously, Australia. Populations of these mosquitoes remain in northern Australia, where malaria was once endemic [1]. The first recorded outbreak of malaria in Australia was in 1843 at Port Essington, Northern Territory [2], although it is likely that the indigenous human population of Australia were exposed to malaria prior to European settlement. Outbreaks continued until the mid 1960s, including the largest Australian civilian outbreak of P. vivax in 1942 at Cairns, Queensland, affecting over 700 people [2, 3]. Anopheles farauti (s.l.), or the An. farauti complex, was the purported primary vector during this epidemic $[4,5]$. Measures implemented to control malaria included modification of mosquito habitats, swamp drainage to kill larvae, pyrethrum spraying to control adult mosquitoes, and the distribution of mosquito nets [6]. Outbreaks of malaria were common until the latter half of the 20th Century; the most recent notable case occurred in 2002 at Noah Beach, close to Cairns, with transmission of $P$. vivax, and where $A n$. farauti (s.s.) was identified as the vector [7]. Therefore, the potential exists for malaria transmission to reoccur in Australia should malaria parasites be introduced by the arrival of infected persons or the transportation of infected mosquitoes, most likely from nearby PNG and the Solomon Islands.

The An. farauti complex is comprised of eight morphologically indistinguishable species, three of which are common in northern Australia: Anopheles farauti (s.s.) Laveran (herein referred to as An. farauti), An. hinesorum Schmidt (herein referred to as $A n$. hinesorum) and $A n$. torresiensis [1]. Although these species are morphologically indistinguishable, they exhibit habitat and behavioural differences. Anopheles farauti is a coastal species and larvae are adapted to brackish water [8-11]. Anopheles hinesorum is also readily found in coastal areas, but is also found inland, including highlands, and is adapted to cooler climates $[1,10,12]$. Accordingly, the two species are sympatric in some but not all locations. Anopheles farauti and An. hinesorum are both anthropophagic in PNG; however, $A n$. hinesorum demonstrates geographical variations in host preferences, being predominantly zoophagic in the Solomon Islands $[4,11]$.

The majority of information regarding $A n$. farauti and $A n$. hinesorum behaviour is derived from studies in PNG and the Solomon Islands, where they are malaria vectors [10, 11, 13-16]. The role of An hinesorum in the transmission of malaria is more complex. Whereas $A n$ hinesorum is a major malaria vector in PNG, limited human feeding occurs in the Solomon Islands with only a single sporozoite-infected specimen collected in the Western
Province [17]. Although there is little evidence that $A n$. hinesorum has been a vector in Queensland, Australia $[10,11]$, its current vector status in PNG and the potential for cold-adaptation to develop, means there is a high priority to study species-specific behaviours of this vector in Australia.

Efforts to control the vector capacity of the An. punctulatus group in the Solomon Islands led to behavioural resistance in $A n$. farauti, effectively shifting host-seeking and blood-feeding from indoor and outdoor all-night biting to earlier in the evening and outdoors $[1,8,18-$ 23]. Such changes in the timing of blood-feeding due to selection imposed by insecticide-treated bednets and/or residual spraying has been reported in a range of anophelines, and may limit the efficacy of bednets as a malaria control strategy [24-28]. While human landing catches have been used to assess biting times in areas subjected to insecticidal selective pressure (e.g. regions of the Solomon Islands, PNG, Indonesia and Vanuatu) and in areas with an absence of vector control pressure, it is unclear whether the underlying pattern of flight activity may have been modified in these mosquito populations. We hypothesize that the flight activity profiles of these mosquitoes will parallel their landing catch/biting profiles and hence timing of blood-feeding. Such information on mosquito behaviour may assist in understanding the mechanism of changes in vector behaviour and contribute to our understanding of how to best control the vector in the future. This is especially important in locations where behavioural shifts in biting have occurred, posing complications for the control of malaria transmission [29].

The expression of 24-hour rhythms in mosquitoes at the molecular, physiological and behavioural levels is driven by an interaction between an endogenous circadian clock and the environmental light-dark (LD) cycle [3036]. Anopheline mosquitoes exhibit predominantly nocturnal patterns of behaviour that include flight activity, swarming and mating, host-seeking/blood-feeding, sugar-feeding, oviposition and pupation [30, 31, 37-40]. Furthermore, many aspects of mosquito biochemistry and physiology are rhythmically regulated, including metabolic detoxification, sensitivity to insecticides, and sensitivity to host odors [34, 38, 41].

Circadian rhythms refer to $\sim 24 \mathrm{~h}$ biological processes driven by an endogenous clock, i.e. they persist under constant conditions. Conversely, diel rhythms are $24 \mathrm{~h}$ rhythms observed under normal environmental LD cycle conditions. Diel rhythms can either be driven directly by the LD cycle or generated through an interaction between the circadian clock and environmental timing cues $[36,42]$.

Mosquitoes of the An. farauti complex are nocturnal, and their diel landing catch/biting patterns are affected 
by environmental factors such as moonlight, as well as by gonotrophic age/parity [8, 11, 43-45]. However, there is only a single study that reports on the timing of $A n$. farauti (s.s.) flight activity, specifically examining its response to differing photoperiods [46, 47]. Although little is known about the diel flight activity patterns of $A n$. farauti complex mosquitoes, it is plausible that they are influenced by season, reproductive state and age, as observed in a range of mosquito species $[8,37,40,48$, 49]. Therefore, exploring the effect of gonotrophic age on diel rhythms in populations of two major malarial vectors may provide useful information that would not be obvious from comparing species alone. Here we report the results of our investigation of diel patterns of spontaneous flight activity comparing the reproductive states of two sympatric species of the An. farauti complex in Australia for differences in their temporal profiles of flight activity.

\section{Methods}

\section{Mosquito collections}

Anopheles farauti (s.s.) and An. hinesorum mosquitoes were collected overnight at the same site in northern Queensland, Australia by $\mathrm{CO}_{2}+$ octenol-baited passive traps [50] near a mangrove forest at McGregor Road and Dunne Road, Smithfield, QLD 4878, Australia (S16 $\left.49^{\prime} 30^{\prime \prime} \mathrm{S}, 145^{\circ} 42^{\prime} 21^{\prime \prime} \mathrm{E}\right)$ at $9 \mathrm{~m}$ above sea level on October 17 and 23, and November 26, 2014. To reduce metabolic stress in caught mosquitoes, honey-impregnated filter paper was provided and high humidity was maintained by a moist sponge set inside the passive trap. Traps were set during the late afternoon and collected early the next morning. Captured adult female mosquitoes were transported to the laboratory and immediately transferred to the locomotor activity-recording device. Species and reproductive state of each mosquito was assessed after recording activity for at least 4 days.

\section{Locomotor activity recording}

Mosquito flight activity was measured with a Locomotor Activity Monitor 25 (LAM 25) system (TriKinetics, Waltham, MA, USA) by placing individual mosquitoes in 25 $\times 150 \mathrm{~mm}$ clear glass tubes with access to $25 \%$ honey or $20 \%$ sucrose provided ad libitum. Flight activity per minute was monitored by recording breaks in a series of infrared beams (Fig. 1a). LAM 25 units were placed in a light-controlled box on a $\sim 12 / 12 \mathrm{~h}$ LD cycle adjusted to local time civil sunset and sunrise $(\sim 10.5 \mathrm{~h}$ full light, $\sim 10.5 \mathrm{~h}$ darkness, and $90 \mathrm{~min}$ dawn and 90 min dusk transitions) with full light in the LAM 25 at 248 lux. Figure 1b shows output of an 'actogram' (activity pattern) for an individual mosquito of each species, plotted on a new line for each $24 \mathrm{~h}$ day. The height of each bar indicates the intensity of mosquito flight activity in each 6 min time bin, i.e. how many beam break events were detected per unit time. Total duration of the 'light' phase of the LD cycle (i.e. duration of full light plus dim light) was set to be equivalent to the ambient conditions when mosquitoes were caught in the field, including twilight, and corroborated from national meteorological information; $13 \mathrm{~h} 45 \mathrm{~min}$ (18th and 24th October collections) and $13 \mathrm{~h} 19 \mathrm{~min}$ (27th November collection). Full light and dawn/dusk transitions were provided by alternate white LED strips (60 per meter; warm white, type 3528 ; and cool white, type 5050) mounted on the lid of the
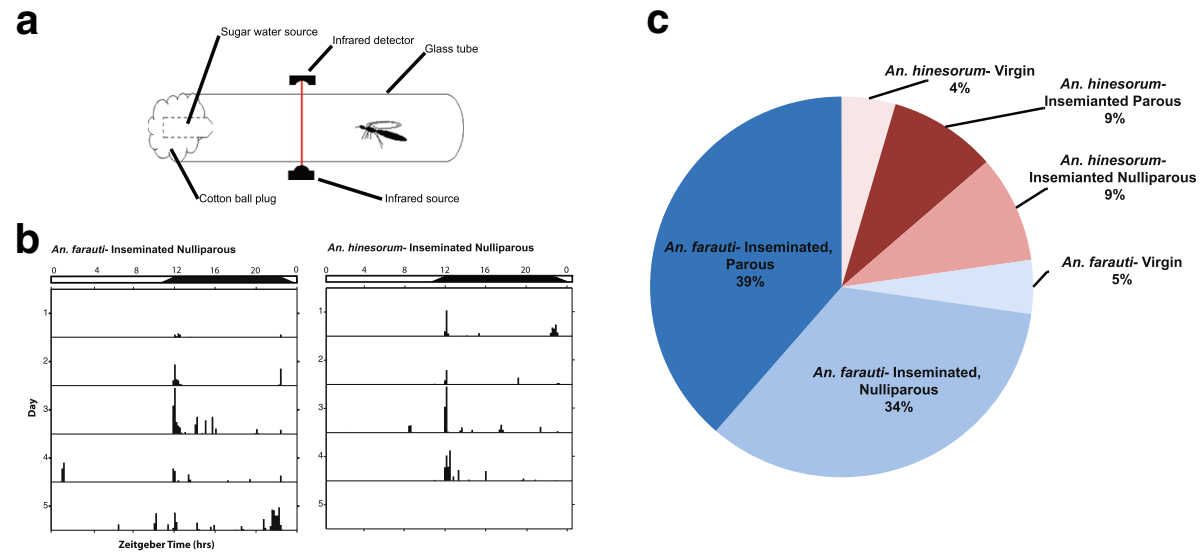

Fig. 1 Behavioural analysis of flight activity in two sympatric species of wild-caught An. farauti complex mosquitoes. a Diagram of LAM25 unit to record individual mosquito activity by continuous infrared beam-break monitoring. b Representative actograms from individual An. farauti and An. hinesorum female mosquitoes. Days are reported on vertical axis and sequential $24 \mathrm{~h}$ periods are shown on the horizontal axis. Zeitgeber time (ZT) with ZT12 being the time of lights off, ZT10.5 the start of the $1.5 \mathrm{~h}$ dusk transition, and ZT0 the end of the $1.5 \mathrm{~h}$ dawn transition and $12 \mathrm{~h}$ after the onset of night. Flight activity presented in 6 min bins. Day and night are indicated by horizontal white/black bars. c Distribution of proportions of experimental mosquitoes by species and reproductive status 
box. Time of day was reported in $24 \mathrm{~h}$ Zeitgeber time (ZT) with ZT12.0 defined as time of lights-off under the LD cycle. The start of the dusk transition was defined as ZT10.5 or ZT10:30. Light intensities at the lower and higher levels of the LAM unit were measured in lux during the dusk cycle: At ZT10:40 light was 69-114 lx; at ZT10:50, 60-100 lx; at ZT11:00, 46-78 lx; at ZT11:10, 33-54 lx; at ZT11:20, 19-31 lx; at ZT11:30, 8.2-14.3 lx; at ZT11:40, 3.1-5.2 lx; at Z11:50, 0.8-1.4 lx; and at ZT11:55, $0.2-0.33 \mathrm{~lx}$. The reverse occurred for the dawn cycle, which started at ZT22:45 and ended at ZT0:15 (full lights on) (October collections) and started at ZT22:19 and ended at ZT23:50 (full lights on) (November collection), and reflects the 26 min shorter ambient photoperiod between collections. The light/humidity-proof box was kept inside a temperature-controlled insectary. The box was provided with cups containing water and a sponge to facilitate a high level of humidity throughout the experiment. Mosquitoes were maintained at $26 \pm 0.5{ }^{\circ} \mathrm{C}$ and 80 $\pm 10 \%$ relative humidity, measured using a $\mathrm{HOBO}$ datalogger (Onset, Bourne, MA, USA). Mosquito flight activity was monitored for 4-5 days, depending upon survivorship of mosquitoes. Locomotor flight activity was viewed in actogram format using ClockLab v.2.61 analysis program (Actimetrics, Wilmette, IL, USA). A total of 101 female mosquitoes were assayed in three separate trials.

\section{Analysis of reproductive state and species}

At the end of the flight activity assays, mosquitoes were removed from the LAM unit. Reproductive organs were dissected from $\mathrm{CO}_{2}$ anesthetized mosquitoes, under a light microscope; spermatheca were examined for spermatozoa, and parity status determined by examining ovaries for the presence (or absence) of tracheole skeins on air-dried slides [51, 52]. Immediately following dissection of a mosquito, the remaining carcass was fixed in $100 \%$ ethanol. Fixed mosquitoes were processed at the University of Queensland for genotyping. Genomic DNA was extracted using a salt extraction method [53] and then identified to species by a species-specific PCR-RFLP of the ITS2 locus [54].

\section{Diel (LD cycle) activity analysis}

Flight activity profiles were created for each species and by reproductive status and parity. Mosquitoes were classified as either inseminated nulliparous, inseminated parous, or virgin. Activity was visualized in one minute and one-hour bins to analyze for time-specific differences between species and between species in each of the reproductive/age categories. For each group, the percent accumulation of daily activity over the 24 hour period was also calculated, starting at the onset of the daytime (ZT0) as $0 \%$.
The average onset of flight activity was determined from the activity data recorded by the LAM 25 unit for each mosquito with at least 3 days of flight activity. In some cases either the first or last days were excluded where behaviour was absent or erratic (this occurred in 26 mosquitoes, resulting in a total of 38 days that were excluded). Activity onset was defined as the first minute followed by two consecutive minutes between ZT11 and ZT13 with flight activity of at least one infrared beam break per minute. In some instances, no such continuous block of activity was recorded and those days $(n=$ 17 days across 15 mosquitoes) were therefore excluded from the determination of the average daily onset for that mosquito. Accordingly, data for each mosquito was derived from a minimum of 3 days and a maximum of 5 days. Maximum peak of flight activity was determined from activity data recorded by the LAM 25 unit for each mosquito. The data were first smoothed into 5 min running averages, and the average peak value across all days ( $\geq 3$ days) for each animal was determined. The maximum peak was defined as the minute between ZT11 and ZT13 with the highest infrared beam breaks per minute. In days where multiple minutes during this period had equally high activity, the first maximum peak that occurred was considered the peak.

The activity data were also smoothed using a $30 \mathrm{~min}$ running average, transformed by Z-score normalization, and analyzed at a $1 \mathrm{~min}$ resolution for differences in activity between species across the $24 \mathrm{~h}$ period. A more focused analysis between ZT11 and ZT13 was also performed in which the data were smoothed using a 5 min running average, Z-scored, and also analyzed at the level of 1 min sampling (i.e. minute-to minute).

\section{Statistical analysis}

Statistical analysis and graphing were performed using Sigma-Plot 12 (Systat Software, Chicago, IL, USA) and GraphPad Prism 5 (Prism, La Jolla, CA, USA). Non-parametric statistical analyses were used when the Shapiro-Wilk normality test failed $(P<0.05)$ or data were cube-root or square-root transformed to correct for non-normal distributions to allow for parametric analysis. For data that passed a Shapiro-Wilk normality test, a one-factor ANOVA or Student's t-test was performed to evaluate differences between groups. For consistency, in comparing the onset times between reproductive states and species-specific reproductive groups data that did not pass normality test, even when square- and cube-roots of values were analyzed, non-parametric tests were utilized. Due to the low capture rate of virgin mosquitoes, virgin female groups were excluded from statistical analyses when based on reproductive state. Statistical significance was at the level of $P<0.05$. 


\section{Results}

Profile of the wild-caught mosquitoes used in the activity analysis

The dominant reproductive status of wild-caught female An. farauti and An. hinesorum was inseminated-nulliparous (Fig. 1a, c). Overall, activity data was obtained for 101 mosquitoes, of which 44 were successfully assessed for both spermatheca status and ovary status. Ninety-one percent of the sampled females were inseminated (spermatozoa positive; $n=40$ ) and 9\% were virgin (spermatozoa negative; $n=4)$, and $48 \%(n=21)$ had previously oviposited (were parous), leaving $43 \%(n=19)$ that were nulliparous. No mosquitoes were identified as gravid. Molecular analysis revealed that 69 (68\%) of anophelines were An. farauti (s.s.) and 32 (32\%) were An. hinesorum. Activity for 3-5 days (recorded as beam break counts) (Fig. 1a) revealed distinct diel nocturnal flight activity (Fig. 1b).

Analysis of flight activity for all mosquitoes collected: Anopheles farauti and An. hinesorum mosquitoes both showed daily nocturnal rhythms of flight activity with a pronounced activity peak during the early night. There were no statistically significant species differences in total counts of flight activity, possibly due to small sample sizes and large variance. However, these trends suggest a higher level of activity in $A n$. hinesorum across all reproductive states compared to An. farauti. Anopheles farauti females $(n=69)$ had a median daily activity of 133 beam break counts per day (counts), while $A n$. hinesorum $(n=32)$ had a median count of 218 (Mann-Whitney test, $\left.U_{(99)}=910.50, P=0.158\right), 85$ counts more than $A n$. farauti.

Analysis of total daily activity for mosquitoes of known gonotrophic state: Inseminated nulliparous An. farauti $(n=15)$ had a median daily activity of 198 counts, while inseminated nulliparous An. hinesorum $(n=4)$ had a median count of 408, 210 counts more than An. farauti $\left(U_{(17)}=17.00, P>0.05\right)$. Inseminated parous $A n$. farauti $(n=17)$ had a median daily activity of 192 counts, while inseminated parous $A n$. hinesorum $(n=4)$ had a median count of 475, 283 more than An. farauti $\left(U_{(19)}=15.00\right.$, $P=0.097)$. Virgin $A n$. farauti $(n=2)$ had a median daily activity of 188 counts, while virgin $A n$. hinesorum ( $n=$ 2) had a median count of 219,31 counts more than $A n$. farauti.

Analysis of differences in the pattern of flight activity across the diel between species: Flight activity data were arranged into 1-hour bins to analyse the hourly pattern of flight across the diel (Figs. 2 and 3). A pronounced species difference in the pattern of activity was noted following the onset of night at ZT12-13 (Fig. 2); although there was no significant main effect of species, the effect of ZT diel time and the interaction between species and time were significant (two-way repeated

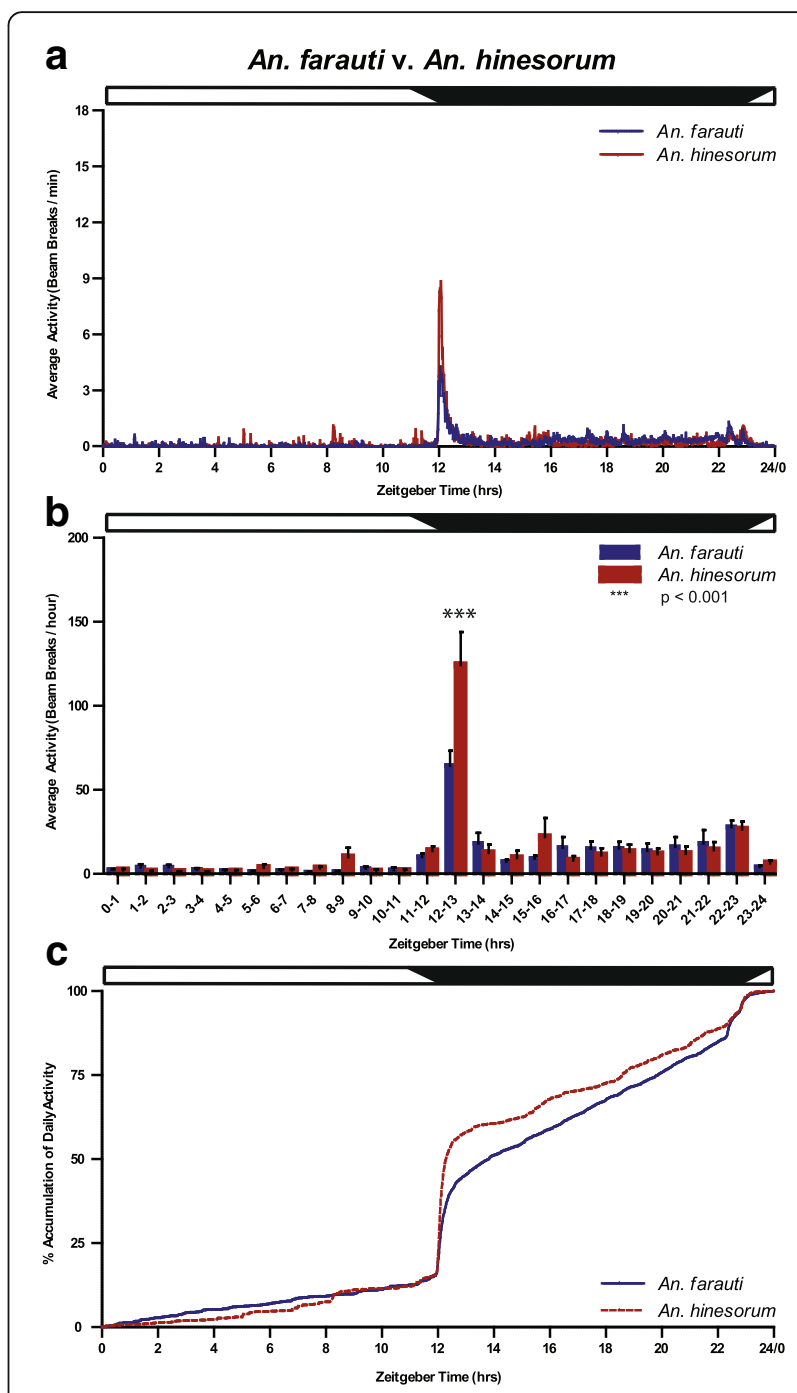

Fig. 2 Average and accumulated activity over the 24 h day in An. farauti and An. hinesorum mosquitoes. Twenty-four hour distribution of mosquito flight activity measured by infrared beam breaks of An. farauti (blue) and An. hinesorum (red) females regardless of reproductive status under LD cycle conditions. a Activity counts per minute (mean \pm SEM). $\mathbf{b}$ Total activity within each hourly time bin (mean \pm SEM). $\mathbf{c}$ Accumulation of daily total mosquito activity. Statistical analysis of accumulation data performed at 50 and $75 \%$ of total daily activity (Additional file 3: Figure S3). Data lines represent average percent measures. Zeitgeber times as for Fig. 1 legend. Day and night are indicated by horizontal white/black bars. Two-factor repeated measures ANOVA was performed followed by Tukey's post-hoc tests, ${ }^{* * *} P<0.001$

measures ANOVA: effect of species, $F_{(1,2039)}=0.87, P=$ 0.354 ; effect of ZT, $F_{(23,2039)}=34.16, P<0.001$; interaction, $F_{(23,2039)}=4.09, P<0.001$; Fig. $\left.2 \mathrm{~b}\right)$. The activity levels in $A n$. hinesorum at ZT12-13 were elevated relative to An. farauti.

Analysis of flight activity with regard to gonotrophic state: a pronounced species difference in flight activity for both nulliparous and parous groups was observed 

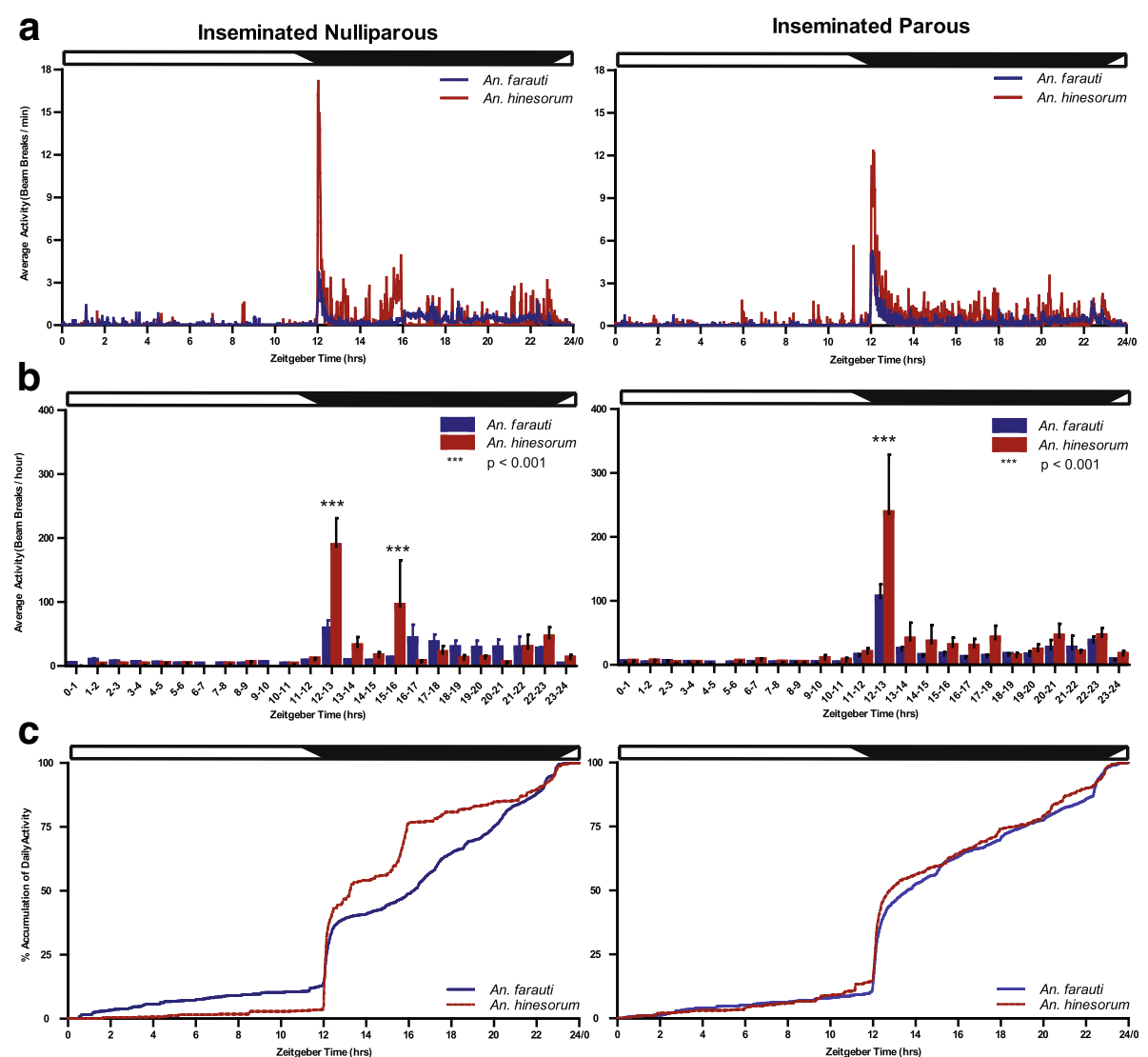

Fig. 3 Flight activity profiles for An. farauti and An. hinesorum mosquitoes according to reproductive status. Twenty-four hour distribution of mosquito flight activity measured by infrared beam breaks of An. farauti (blue) and An. hinesorum (red) females of each reproductive state under LD cycle conditions at $\mathbf{a}$ minute and $\mathbf{b}$ hour resolutions. Hour resolutions are mean \pm SEM of total activity within each hourly time bin. $\mathbf{c}$ The accumulation dynamics of daily total mosquito activity. Zeitgeber times as for Fig. 1 legend. Day and night are indicated by horizontal white/ black bars. Two-factor repeated measures ANOVA was performed followed by Tukey's post-hoc tests, ${ }^{* * *} P<0.001$

during early night (ZT12-13), and additionally in the inseminated nulliparous group, a species difference was detected $4 \mathrm{~h}$ into the scotophase (ZT15-16) (nulliparous: species, $F_{(1,455)}=0.83, P=0.375 ; \mathrm{ZT}, F_{(23,455)}=$ 6.99, $P<0.001$; interaction, $F_{(23,455)}=2.99, P<0.001$; parous: species, $F_{(1,502)}=3.08, P=0.095$; ZT, $F_{(23,502)}=$ 14.60, $P<0.001$; interaction, $F_{(23,502)}=2.28, P<0.001$; Fig. $3 \mathrm{~b})$. At these two specific times of the night, the activity levels in $A n$. hinesorum were elevated relative to An. farauti.

When the data were examined to specifically compare reproductive states within each species, higher levels of activity were detected in parous An. farauti at ZT12-13. Conversely higher levels were observed in nulliparous $A n$. farauti during the middle of the scotophase at ZT16-17 (reproductive state, $F_{(1,767)}=0.00, P=0.958$; $\mathrm{ZT}, F_{(23,767)}=9.40, P<0.001$; interaction, $F_{(23,767)}=$ $1.58, P=0.042$; Additional file 1: Figure S1b). However, no significant differences were observed between reproductive groups for An. hinesorum (reproductive state, $F_{(1,191)}=0.43, P=0.535 ; \mathrm{ZT}, F_{(23,191)}=9.15, P<0.001$; interaction, $F_{(23,191)}=0.50, P=0.973$; Additional file 1: Figure S1b).

Comparison of the two species using the smaller cohort of mosquitoes for which insemination and parity could be determined revealed a pronounced species difference at ZT15-16 as well as at ZT12-13 (species, $F_{(1,1055)}=2.54, P$ $=0.119 ; \mathrm{ZT}, F_{(23,1055)}=22.33, P<0.001$; interaction, $F_{(23,1055)}=4.30, P<0.001$; Additional file 2: Figure S2). While the higher activity seen for An. hinesourum at ZT12-13 is consistent with the larger cohort of mosquitoes (Fig. 2), the higher activity at ZT15-16 is a feature consistent with the nulliparous subgroup (Fig. 3).

The pattern of accumulated flight activity over $24 \mathrm{~h}$ (measured over 3-5 days) was compared between the two species to determine when mosquitoes had accumulated a given amount of their daily total flight activity (Figs. 2c and 3c). A significant species difference was found in percent accumulation at the time at which $A n$. farauti and An. hinesorum reached $75 \%$ accumulation of activity $\left(U_{(83)}=555.00, P=0.031\right)$, with $A n$. hinesorum reaching this point earlier. Anopheles hinesorum shows 
similar accumulation over the light phase of the LD cycle (day) (ZT0 to ZT12), but differentiates during the dark phase of the cycle (scotophase) (Fig. 2c). While not significant, the $A n$. hinesorum mean value also reached $50 \%$ accumulation of activity before An. farauti $\left(t_{(83)}=\right.$ $0.747, P=0.457$ ) (Additional file 3: Figure S3). Furthermore, no significant reproductive state differences were detected at these two accumulation thresholds (Fig. 3c).

\section{Times of onset and maximum peak of dusk/early night flight activity}

Species- or reproductive state-specific differences in the time of activity onset and the maximum peak of elevated dusk-related flight activity, key features of the diel activity profile, were investigated. A significant difference was revealed in activity onset times between $A n$. farauti and $A n$. hinesorum $\left(U_{(83)}=547.00, P=0.033\right)$ (Fig. 4a). Anopheles farauti commenced activity at a median time of ZT12:01, while $A n$. hinesorum activity onset was 3 min earlier at a median time of ZT11:58. The mean onset of the An. farauti mosquitoes was ZT12:01, while the An. hinesorum mean activity onset commenced $4.8 \mathrm{~min}$ earlier at ZT11:56 (Table 1). No significant differences were observed in the time of activity onset among reproductive state groups (inseminated parous versus inseminated nulliparous, $\left.U_{(38)}=158.0, P=0.268\right)$ or species-specific reproductive state groups (Kruskal-Wallis one-way ANOVA, $H_{(3)}=3.98, P=0.264$ ) (Additional file 4: Figure S4a, Tables 2 and 3).

A significant difference between $A n$. farauti and $A n$. hinesorum maximum peak in flight activity per minute
Table 1 Mean \pm SEM in Zeitgeber time (ZT) for onset of activity and maximum peak values in An. farauti and An. hinesorum mosquitoes

\begin{tabular}{lll}
\hline Species & $\begin{array}{l}\text { Anopheles farauti } \\
(n=58)\end{array}$ & $\begin{array}{l}\text { Anopheles hinesorum } \\
(n=27)\end{array}$ \\
\hline Onset (ZT) & $12: 01 \pm 0: 01$ & $11: 56 \pm 0: 02$ \\
Maximum peak (ZT) & $12: 09 \pm 0: 01$ & $12: 05 \pm 0: 02$ \\
\hline
\end{tabular}

(with a 5 min running average) between ZT11 and ZT13 was found $\left(U_{(83)}=565.5, P=0.040\right)$ (Fig. 4b). The median peak time in An. farauti occurred at ZT12:07, while the median peak time in An. hinesorum was 3 min earlier at ZT12:04. Similarly, the mean peaks for An. farauti and An. hinesorum were at ZT12:09 and ZT12:05, respectively, revealing a 4.2 min difference (Table 1). Significant differences were not found for the time of the maximum peak between reproductive states (inseminated parous versus inseminated nulliparous, $U_{(38)}=$ 187.5, $P=0.753)$ or species-specific reproductive state groups $\left(H_{(3)}=1.24, P=0.743\right)$ (Additional file 4: Figure S4b, Tables 2 and 3). The earlier onset and earlier maximum peak of activity in An. hinesorum can be seen in a Z-scored plot of two individual representative mosquitoes (Fig. 4c). See Tables 1, 2 and 3 for species and reproductive group-specific mean \pm SEM activity onset and maximum peak values.

To determine if the dusk/early night related flight activity differed between $A n$. farauti and An. hinesorum in their temporal dynamics, we analyzed the build-up and decline of daily activity with the activity of each
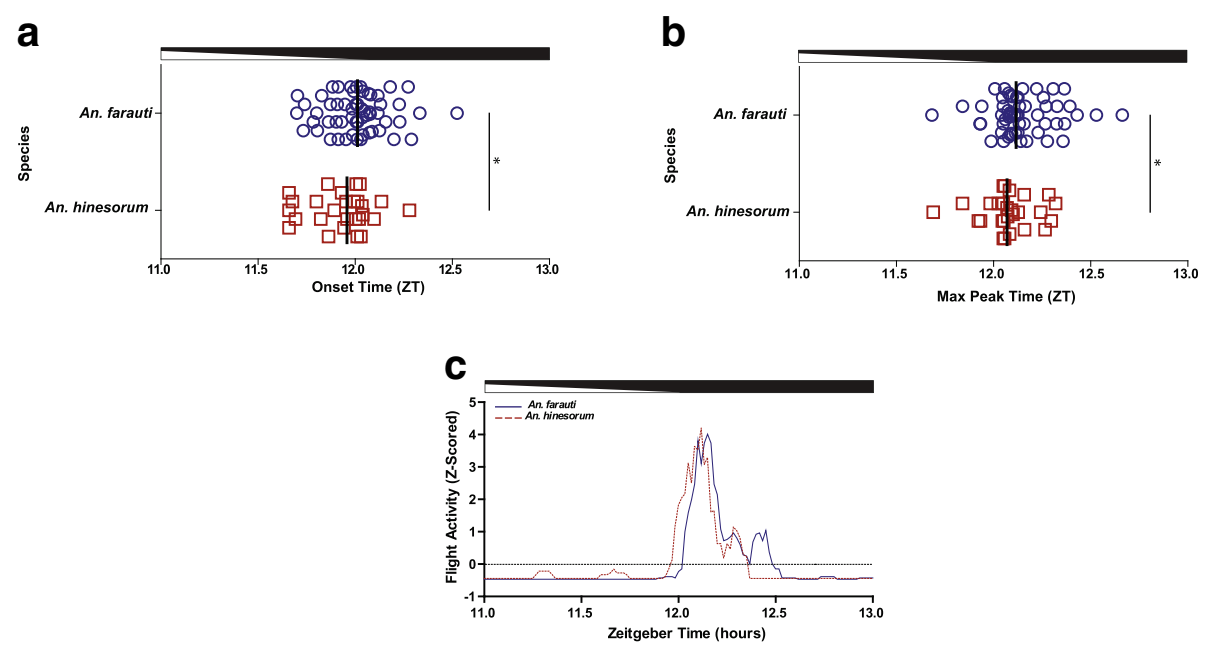

Fig. 4 Onset and peak of dusk/early night-related flight activity in An. farauti and An. hinesorum mosquitoes. Differences between An. farauti (blue) and An. hinesorum (red) in time of dusk related onset of flight activity and peak of flight activity: $\mathbf{a}$ time of onset of activity, $\mathbf{b}$ time of peak of activity, and $\mathbf{c}$ a representative Z-score plot of activity. Median values (lines) and individual mosquitoes (An. farauti, circles; An. hinesorum, squares) in Zeitgeber time (ZT, h). Dusk transitions are indicated by horizontal white/black bars. Mann-Whitney U-tests were performed, ${ }^{*} P<0.05$. c Representative activity plots for individual mosquitoes from each species, demonstrating a delayed onset and delayed peak of activity for An. farauti compared to An. hinesorum 
Table 2 Mean \pm SEM in Zeitgeber time (ZT) for onset of activity and maximum peak values for both An. farauti and An. hinesorum combined and comparing three reproductive states

\begin{tabular}{llll}
\hline Reproductive state & $\begin{array}{l}\text { Inseminated, parous } \\
(n=21)\end{array}$ & $\begin{array}{l}\text { Inseminated, nulliparous } \\
(n=19)\end{array}$ & $\begin{array}{l}\text { Virgin } \\
(n=4)\end{array}$ \\
\hline Onset (ZT) & $11: 58 \pm 0.02$ & $12: 02 \pm 0: 02$ & $12: 02 \pm 0: 03$ \\
Maximum peak (ZT) & $12: 07 \pm 0: 01$ & $12: 09 \pm 0: 02$ & $12: 12 \pm 0.04$ \\
\hline
\end{tabular}

individual animal first smoothed to $30 \mathrm{~min}$ and Z-scored; species differences were reported when $\geq 10$ consecutive minutes were significantly different. As shown in Fig. $5 \mathrm{a}$, there is a relative increase in mosquito activity around the time of lights-off (i.e. ZT12:00) between ZT11:55 to ZT12:15 in An. hinesorum $(n=27)$ relative to An. farauti $(n=58)$ (t-tests, $P<0.01)$. Additionally, there was a significant decrease in mosquito activity after the peak of activity between ZT13:39 and ZT14:09 (t-tests, $P<0.01$ ). Furthermore, An. farauti showed a relative increase in activity later in the night between ZT22:09 and ZT22:39 (t-tests, ZT22:09-ZT22:18, $P<0.01$; ZT22:19-ZT22:39, $P<0.05)$.

In addition to the $24 \mathrm{~h}$ analysis of behaviour, a targeted analysis of dusk/early night associated activity between ZT11 and ZT13 was examined as this is the expected time of onset of behaviours related to migration, sugar-feeding, host-seeking, and in the case of virgins and gravids, respective mating and oviposition activities. The flight activity of each individual animal was smoothed to $5 \mathrm{~min}$ and Z-scored. Figure $5 \mathrm{~b}$ shows consecutive minutes of significance. Anopheles hinesorum showed a relative increase of activity compared to An. farauti from ZT12:03-ZT12:05 (t-tests, ZT12:03ZT12:04, $P<0.01$; ZT12:05, $P<0.05)$. After the peak of activity, $A n$. hinesorum showed a decrease in activity relative to An. farauti from ZT12:33 to ZT12:39 (t-tests, ZT12:35-ZT12:38, P < 0.01; ZT12:33-ZT12:34, ZT12:39, $P<0.05)$. Analysis of mosquitoes arranged by reproductive group and by both species and reproductive group yielded no significant differences.

\section{Discussion}

The flight activity patterns of two sympatric and cryptic species of the An. farauti complex, An. farauti (s.s.) and An. hinesorum, in northern Queensland, Australia were characterized. We investigated the potential temporal differences in behaviour between the species, including total daily activity, accumulation dynamics, and dusk/ early night-related flight activity, and their relationship to reproductive state. Both An. farauti and An. hinesorum exhibited daily nocturnal rhythms of flight activity with a pronounced peak at the onset of night at the end of dusk, and a smaller but obvious peak towards the end of night at dawn. During the remainder of the scotophase, intermittent and often lower level activity was observed. This general profile of $24 \mathrm{~h}$ activity is consistent with observations described previously in adult anopheline mosquitoes, including An. farauti [35, 37, 47, 55]. Such 'bimodal' activity patterns are preserved under constant dark conditions, indicating the underlying circadian clock contributes to the regulation of the behaviour. Furthermore, the bout of intense activity observed occurring at dusk/early night (ZT12-13) is relatively short-lived, being less than $45 \mathrm{~min}$, and matches the length of a similar dusk bout of activity documented in the anopheline mosquitoes in the An. gambiae complex and in An. stephensi $[8,35,37,40,49,56]$.

Diel analysis of flight activity behaviour in the An. farauti complex has thus far been limited to a single study of adult female $A n$. farauti Laveran [presumed An. farauti (s.s.)], examined under differing photoperiods [47]. The study by Taylor [47] differs considerably from the present analysis, as it used acoustic recordings to assay flight activity, exposed mosquitoes to abrupt transitions from light to dark and dark to light, and the temporal resolution of analysis was relatively large ( 1 hour bins only) [47]. Furthermore, it is unclear whether the subjects studied were captured as adults or reared from larvae. In the present study, we attempted to tease apart potential species- and reproductive stage/age-specific differences for two species in the An. farauti complex. Wild-caught female mosquitoes were subjected to a LD cycle with dawn and dusk transitions, recorded beam-break activity by minute, and

Table 3 Mean \pm SEM in Zeitgeber time (ZT) for onset of activity and maximum peak values for An. farauti and An. hinesorum and across three reproductive states

\begin{tabular}{lllllllll}
\hline Species & \multicolumn{2}{l}{ Anopheles farauti } & & & Anopheles hinesorum & \\
\cline { 2 - 3 } Reproductive state & Inseminated, parous & Inseminated, nulliparous & Virgin & & & Inseminated, parous & Inseminated, nulliparous & Virgin \\
& $(n=17)$ & $(n=15)$ & $(n=2)$ & & $(n=4)$ & $(n=4)$ & $(n=2)$ \\
\hline Onset (ZT) & $11: 58 \pm 0: 02$ & $12: 04 \pm 0: 03$ & $12: 07 \pm 0: 04$ & $11: 57 \pm 0: 05$ & $11: 53 \pm 0: 05$ & $11: 59 \pm 0.03$ \\
Maximum peak (ZT) & $12: 06 \pm 0: 01$ & $12: 09 \pm 0: 04$ & $12: 12 \pm 0: 07$ & $12: 11 \pm 0: 04$ & $12: 09 \pm 0: 04$ & $12: 12 \pm 0: 07$ \\
\hline
\end{tabular}




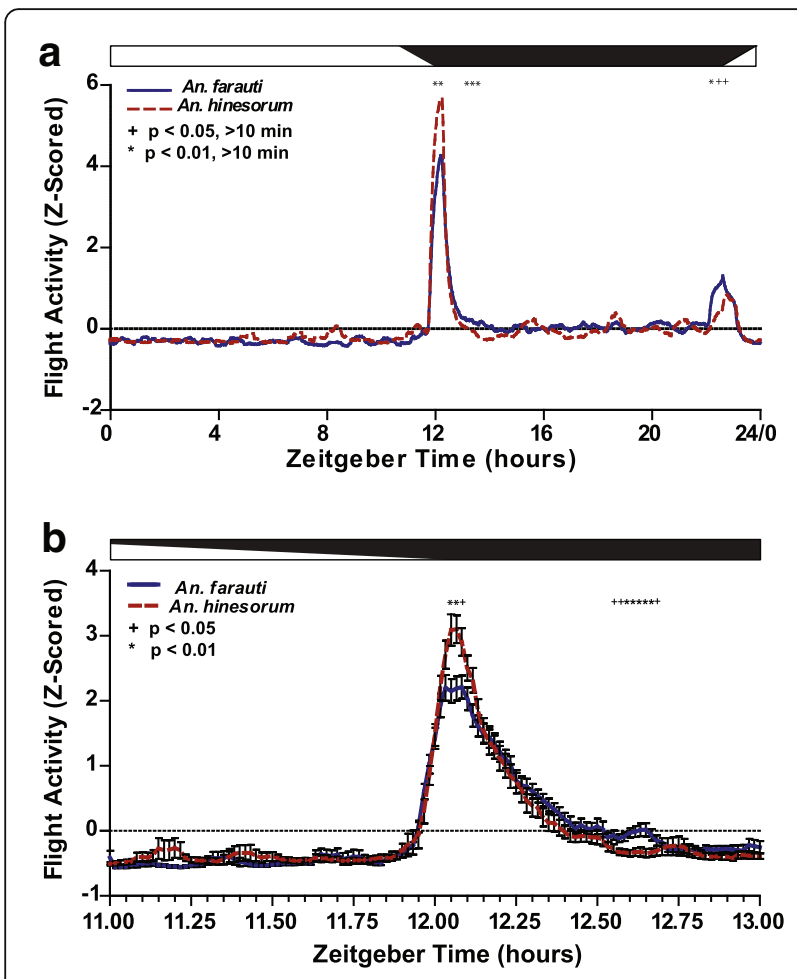

Fig. 5 Z-scored flight activity shows multiple time differences between An. farauti and An. hinesorum mosquitoes at the onset of night and during other parts of the scotophase. a Flight activity of An. farauti (blue) and An. hinesorum (red) over $24 \mathrm{~h}$ under normal LD conditions. Values are mean of Z-scored activity. Day and night are indicated by horizontal white/black bars. b Dusk and early night-related flight activity. Values are mean \pm SEM of Z-scored activity between ZT11 and ZT13 under normal LD cycle conditions. Time shown in Zeitgeber time (ZT) decimal format. Dusk progression shown by horizontal white/ black bar. Student's t-tests were performed, $+P<0.05,{ }^{*} P<0.01$

analysed data by hourly and minute-to-minute resolution, thereby allowing for a precise temporal evaluation of behaviour [35].

When the accumulation of flight activity is measured from the start of daytime (ZT0), activity accumulates more rapidly in An. hinesorum than An. farauti. This separation becomes obvious shortly after the onset of night at ZT12, and when analyzed specifically at $75 \%$ accumulation, we see a clear significant difference. This difference is mostly due to the higher level of relative activity expressed by $A n$. hinesorum compared to An. farauti in the dusk/early night-related bout of intense activity ( ZT12-13). The raw counts within this 1-h time bin show $A n$. hinesorum exhibiting levels of activity twice as large as those by $A n$. farauti. Interestingly, when we compare dusk/early night flight activity, the higher level of activity occurred for a duration of $\sim 20$ min between ZT11:55 and ZT12:15. The lower level of activity, as measured in raw counts or following Z-scored normalization, that occurs later and intermittently throughout the remainder of the scotophase/night is comparable between the two species. This focuses our attention on the dusk/ early night related activity and that may relate to one or a combination of natural behaviours observed in An. farauti complex mosquitoes, such as host-seeking, and behaviours reported in other anophelines, such as migration, sugar-feeding, egg laying in gravids, and in virgins, swarming and mating. This differential activity pattern may represent an aspect of behaviour(s) that is elevated in $A n$. hinesorum, e.g. increased feeding activity and/or longer distance/duration of flight.

Anopheles hinesorum began its nocturnal activity 5 min earlier on average than $A n$. farauti, and the maximum peak of activity in An. hinesorum was observed to occur 4 min earlier than for An. farauti. This apparent 'shift' in the activity profile is of particular interest because the differences occur close to ZT12, the onset of night. The intense level and subsequent peak of activity occurring during the early night is the most dominant and consistent feature of the diel profile. This distinct profile may represent a combination of behavioural drives, many of which have been studied in anophelines and in particular members of the An. gambiae complex. These include migration, sugar-feeding, host-seeking and biting, all of which commence during twilight and the onset of night [21, 56-59].

As the majority of captured mosquitoes in the study were identified as either nulliparous or parous females, and few were scored as virgin and none scored as gravid, this makes swarming/mating and oviposition unlikely behaviours to be represented in the underlying flight activity drives of mosquitoes observed here. However, swarming and mating in other anophelines occurs during a small temporal window during dusk [60-62], and peak ovipositioning occurs during the first hour of the night $[37,39,63]$, both of which are coincident with the peak in flight activity of An. farauti complex mosquitoes in the present study.

Due to the reproductive states of the majority of mosquitoes in the study, it is more likely that the intense early nocturnal activity in the flight profile in part reflects other factors, such as movement from resting sites, i.e. daily migration. In An. gambiae, dusk/early night is the time when females depart from their daytime resting sites [57-59]. Furthermore, the flight range for An. farauti has been estimated to be as much as $1.6 \mathrm{~km}$ [64].

Sugar-feeding activity in An. gambiae occurs almost exclusively at night and during the dusk/dawn transitions [56]. However, the expression of this diel pattern is dependent upon the reproductive state of the female [56]. Under laboratory conditions, in inseminated An. gambiae females that were either prevented from blood-feeding or blood-fed but prevented from ovipositioning, a high level 
of sugar-feeding activity was observed during the scotophase and with a noticeable peak during late dusk and early scotophase. Therefore, in the present study, it is possible that the nocturnal flight activity profiles of $A n$. farauti complex females, and especially the dusk/early night peak, reflects this characteristic sugar-feeding behaviour.

Finally, host-seeking behaviour and the propensity to bite are also likely contributing drives underlying the diel flight activity profiles of An. farauti and An. hinesorum. Studies of $A n$. farauti and to a lesser degree An. hinesorum host-seeking behaviour have been undertaken in the Solomon Islands and PNG, primarily using human landing catch (HLC) assays. In many locales, the nocturnal biting profiles for An. farauti consistently show a majority of feeding early in the night. For example, in the Solomon Islands (Haleta, Central Province), $82 \%$ of mosquitoes were captured between 18:00 and 21:00 h, and only $18 \%$ between midnight and 06:00 h [22]. A major peak occurred at 19:00-20:00 h, declining thereafter, and then a small increase in capture rate occurred at the end of the night/dawn between 05:00-06:00 $\mathrm{h}$ [21]. In Honiara, Solomon Islands, as much as $84 \%$ of An. farauti mosquitoes captured by HLCs was during the first hour following sunset [9]. However, in comparison in PNG (Mirap, Madang Province) the shape of the profile is different: an early night peak remains, although a secondary peak occurred between 23:00 $\mathrm{h}$ and midnight, i.e. during the middle of the night [21].

A single study reports on the outdoor biting cycle of An. hinesorum as compared to An. farauti in PNG (Madang and East Sepik provinces) [10]. While An. hinesorum exhibited an early biting profile, with $66 \%$ activity occurring between 18:00 and 22:00 h, An. farauti (s.s.) exhibited a uniform biting profile throughout the entire night and with no obvious peaks. Clearly this study reveals differences in the biting cycle between the two species, as well as An. hinesorum in this locale being an early night biter.

There are three major factors thought to contribute to the shape of the An. farauti host-seeking biting profile, namely long-term exposure to insecticide, outdoor or indoor location of the host and the lunar cycle. In areas where An. farauti (s.s.) populations have been exposed to residual spraying of DDT and/or introduction of insecticidal treated bednets, mosquitoes show significant shifts in their biting profiles, developing an exaggerated peak in biting during the onset of night, as well as increasing the number of outdoor versus indoor biting encounters $[1,8,18-23]$. For example, in the Solomon Islands (Baunasughu, Manibwenta and Maniparegho) prior to exposure to insecticide, indoor biting profiles showed a more uniform pattern during the first half of the night or show peak biting at midnight [20]. Outdoor biting prior to insecticide exposure still showed a peak close to the onset of the night and highest levels of biting occurred during the first half of the night. Following exposure, both indoor and outdoor patterns became similar, with as much as $45 \%$ of nocturnal biting events occurring at dusk and the onset of night (18:30-19:30 h) [20]. Finally, moonlight can impact the nocturnal biting cycle of An. farauti [8]. In PNG (Maraga, Madang Province), the biting cycle of An. farauti showed an initial peak at the onset of night at all times of the lunar cycle. However, on moonless nights, this initial peak was most prominent, followed by a secondary peak occurring before midnight. Under a waxing moon, activity was concentrated in the first half of the night; under a waning moon activity was also highest during the first half of the night, but activity during the second half of the night remained relatively high compared to other phases of the moon. During a full moon, peak biting occurred in the middle of the night.

Relating the natural biting cycles of An. farauti and $A n$. hinesorum is obviously complex due to these differing environmental factors. However, the location in Queensland, Australia where the mosquitoes were captured is not an area with known exposure to insecticides, mosquitoes were captured outdoors, and the conditions in the experimental chamber when assayed for flight activity were 0 lux at night (i.e. the equivalent to a moonless night). Based on the studies from the Solomon Islands and PNG, and under these environmental conditions in Australia, we might predict that the An. farauti (s.s.) mosquitoes would express a diel biting profile with a major peak occurring at the end of dusk into the early night, followed by continued lower level host-seeking activity during the first half of the night. In fact, a recent study in northern Queensland reveals An. farauti (s.s.) during the dry season as exhibiting early night peak biting. Biting was greatest at the onset of night, and while rates declined throughout the scotophase, a secondary peak occurred at 21:00-22:00 $\mathrm{h}$ followed by a small increase occurring near to dawn (W. K. Chow, R. D. Cooper, L. Rigby, P. Pickering, M. Lokhorst and N. Beebe, personal communication). Clearly, there is a correlative relationship between the diel cycles of flight activity and host-seeking activity for An. farauti (s.s.) in Queensland. This includes the intermittent flight activity observed during the scotophase, but especially so at the onset of night when peak outdoor biting occurs, and towards the end of night/dawn when there is a small but distinct rise in both activities. Therefore, it is likely that the diel flight activity profile reflects components of the drive to host seek and blood-feed.

In An. farauti there were differences in the flight activity profiles between nulliparous and parous mosquitoes: activity at the onset of night (ZT12-13) was higher in parous group, while conversely, activity was greater during 
the middle of the night (ZT16-17) in the nulliparous population. It is unclear what this finding means in terms of behavioural drives that may be different between these groups, except that the parous population is predicted to be older. There are known differences in the biting profiles of anophelines, where parous females tend to bite later than nulliparous females [65-68]. However, this phenomenon observed in the field may be associated with gravids ovipositing early in the night, thereby delaying host-seeking activity during the same night. However, it is unclear whether the differences observed in the flight activity profiles between the reproductive states/ages represent intrinsic differences in biting activity.

Thirty minutes after lights off at ZT12.5, An. farauti exhibited a small but significant additional elevation of activity compared to An. hinesorum for $8 \mathrm{~min}$. While the meaning of this differential species activity in terms of natural behaviour is unknown, a similar differential pattern of behaviour was documented for An. gambiae and An. coluzzii males [35]. Here, the two An. gambiae complex species show a separation in their pattern of activity in both the rise in activity after activity onset occurs during dusk, and on the decline of activity following its peak shorty after the onset of night. In that study the authors speculate that this differential pattern of activity represents either earlier swarm-assembly behaviour, or some aspect of pre/postswarming behaviour(s), e.g. increased sugar-feeding activity and/or longer distance/duration of flight. This hypothesis may equally apply to An. hinesorum and An. farauti.

Another bout of differential activity was observed approximately an hour after lights off at $\sim \mathrm{ZT} 13$ and for a longer duration of time (for $\leq 30 \mathrm{~min}$ ). As this occurs in the scotophase well after the dusk/onset of night-related high intensity bout of activity, this 'event' may underlie a natural behaviour distinct from those associated with dusk and the onset of night.

When reproductive status/age is considered, the enhanced level of flight activity at dusk/onset of night in An. hinesorum versus An. farauti was conserved in both groups, namely inseminated nulliparous and inseminated parous. Furthermore, a distinct species difference was observed in the inseminated nulliparous group during the middle of the scotophase/night (ZT15-16), in which An. hinesorum mosquitoes had a pronounced elevated bout of activity. This revealed a conspicuous spike amongst the relatively low level activity recorded during the remainder of the scotophase. In An. hinesorum, flight activity during this hour was not only considerably higher compared to An. farauti but was relatively high compared to almost all times of the night excluding the dusk peak. Interestingly, this elevation of flight activity among inseminated nulliparous An. hinesorum at ZT1516 is consistent with a previous study of HLAs of $A n$. farauti complex mosquitoes in PNG (Madang and East
Sepik provinces) [10]. In this study, biting-landing catches in An. hinesorum were higher during the one hour in the middle of the night at midnight as compared to the preceding two hours and proceeding four hours. This variable pattern of landing catches was not observed for An. farauti, which was more uniformly distributed during the night. It is unclear whether this is simply a coincidence or whether the flight activity pattern observed here is associated with host-seeking behaviour and subsequent human encounters.

These behavioural data provide novel insights into the temporal regulation of flight activity that are important for ongoing efforts to understand An. farauti complex mosquitoes in Australia, the Solomon Islands, PNG, Indonesia and Vanuatu. Historically, northern Australia was malaria-endemic and, while now eliminated, there remains a risk of re-introduction and local transmission due to presence of An. farauti, and possibly An. hinesorum. As recent as 2002 there was an outbreak of $P$. vivax transmission in the Cairns area of Northern Queensland, illustrating the potential for local malaria transmission following introduction of the malaria parasite by infected people. Thus, the An. farauti complex poses a plausible risk for malaria transmission as Australia is surrounded by countries where malaria is endemic $[1,7]$. These data also contribute to our understanding of An. farauti complex mosquitoes in malaria endemic areas, including the Solomon Islands and Papua New Guinea, where as much as $6 \%$ of the human population is infected with $P$. falciparum, $P$. vivax, or $P$. malariae $[14,15]$. Furthermore, as the Queensland populations of An. farauti and An. hinesorum are not routinely exposed to insecticides, these behavioural data can serve as a baseline for comparison with mosquitoes from the Solomon Islands, where the timing of nocturnal biting activity has been shaped by continuing insecticide selective pressure (from insecticide treated bednets and/ or residual spraying) $[11,18,20]$. Although An. farauti and $A n$. hinesorum are morphologically indistinguishable species and are known to reside in slightly different habitats, differences in the timing of their flight behaviour, as proposed in this paper, and in host-seeking behaviour $[10,11]$, make vector control complicated in the areas in which the two species coexist. Understanding the causative factors underlying differences in flight activity may have real implications for controlling these two species, particularly as the number of recommended interventions expands.

The shifts in An. farauti complex biting activity from the middle of the night to earlier in the evening following insecticidal pressure (from insecticide treated bednets and/or indoor residual spraying of insecticides) [20-22], is a function of changes in the flight activity patterns of the mosquitoes, especially for host-seeking 
females. Further analysis of the flight activity of wild populations of An. farauti and An. hinesorum that have been exposed to long-term insecticide pressure would allow for this hypothesis to be tested. Such populations exist in regions of the Solomon Islands in particular. Therefore, the present analysis may provide a baseline for comparing populations of species in the An. farauti complex.

There are certain limitations to the interpretation of these data as the experimental conditions do not represent true unconstrained flight conditions in the wild. The absence of males and insufficient numbers of virgin females in this study limit our understanding of potential species differences in the timing of mating and swarming activity. In other anophelines, it is the males that first assemble the swarm around dusk into which virgin females then enter and mate [37, 69], and it has been proposed that differential timing of swarming might contribute in part to species-specific segregation of mating in sympatric $A n$. gambiae complex mosquitoes [35, 70, 71]. Therefore, the timing of male activity may be more relevant in the context of temporal control of intraspecific mating [35]. However, these data do provide some insight into the precise timing of aspects of flight behaviour in wild populations of predominately inseminated host-seeking mosquitoes (versus laboratory-raised colonies). It is therefore possible that additional but subtle differences exist between the two species and reproductive/age categories.

\section{Conclusions}

In conclusion, in this study of An. farauti and An. hinesorum mosquitoes from northern Queensland, Australia, species and gonotrophic-age related differences were revealed in their circadian/diel-driven flight behaviour. The dusk/early night-related flight activity in An. hinesorum females start and peak earlier than in An. farauti. The shape of the dusk/early night flight activity also differs between species, with An. hinesorum exhibiting a higher level of activity at its peak around ZT12-ZT12.25, and at its decline An. farauti exhibited a small elevation of activity later in the night at $\sim$ ZT12.5. Then one hour into the night at $\sim$ ZT13, the two species show a small divergence in activity. We hypothesize that this differential pattern of activity observed during late dusk/early night represents speciesspecific differences in the timing and pattern of host-seeking behaviour, sugar-feeding activity, and/or distance/duration of migratory flight. Finally, we observed a peak in activity during the middle of the night at ZT15-16 in the An. hinesorum inseminated nulliparous population, which may be related to increased host-seeking behaviour. As $A n$. farauti and An. hinesorum are major malaria vectors from Indonesia through PNG and the Solomon Islands to Vanuatu, these data may prove useful in an improved understanding of mosquito biology that impacts the control of malaria.

\section{Additional files}

\begin{abstract}
Additional file 1: Figure S1. Twenty-four hour distribution of mosquito flight activity measured by infrared beam breaks of a An. farauti and $\mathbf{d}$ An. hinesorum females of the inseminated parous and inseminated nulliparous reproductive states under LD cycle conditions at minute resolution. A similar distribution shows $\mathbf{b}$ An. farauti and e An. hinesorum activity at hour resolution. Accumulation of mosquito activity over a $24 \mathrm{~h}$ time period of $\mathbf{c} A n$. farauti and $\mathbf{f} A n$. hinesorum females was calculated in a similar manner. Zeitgeber time (ZT) with ZT12 being the time of lights off, ZT10.5 the start of the $1.5 \mathrm{~h}$ dusk transition, and ZT0 occurring at the end of the $1.5 \mathrm{~h}$ dawn transition and $12 \mathrm{~h}$ after the onset of night. Day and night are indicated by horizontal white/black bars. (PDF 401 kb)
\end{abstract}

Additional file 2: Figure S2. Twenty-four hour distribution of mosquito flight activity measured by infrared beam breaks of An. farauti and An. hinesorum female regardless of reproductive state, so long as a reproductive state could be determined, under LD cycle conditions at a minute and $\mathbf{b}$ hour resolution. c Accumulation of mosquito activity over a $24 \mathrm{~h}$ time period of An. farauti and An. hinesorum females was calculated in a similar manner. Zeitgeber times as for Fig. 1 and Additional file 1: Figure S1 legends. Day and night are indicated by horizontal white/black bars. (PDF $245 \mathrm{~kb}$ )

Additional file 3: Figure S3. Analysis of the accumulation of flight activity over $24 \mathrm{~h}$ reveals differences between An. farauti complex mosquito species. Zeitgeber time at which each individual mosquito reached a 50\% and $\mathbf{b} 75 \%$ accumulation of activity revealed a species-specific difference. Mean \pm SEM (lines) and individual mosquito time points marking either 50 or $75 \%$ accumulation of individual daily activity (h) (An. farauti, blue circles; An. hinesorum, red squares). Mann-Whitney U-test was performed, ${ }^{*} P<0.05$. (PDF $177 \mathrm{~kb}$ )

Additional file 4: Figure S4. Analysis of the timing of dusk/early night related onset of flight activity and peak of flight activity in An. farauti complex mosquitoes according to reproductive state and species. a Time of onset of activity, and $\mathbf{b}$ time of peak of activity. Median values (lines) and individual mosquitoes (An. farauti, circles; An. hinesorum, squares) in Zeitgeber time $(Z T, h)$. Dusk transitions are indicated by horizontal white/ black bars. (PDF $112 \mathrm{~kb}$ )

\section{Abbreviations}

ZT: Zeitgeber time; LD: light:dark; DD: dark:dark; LAM: locomotion activity monitor

\section{Acknowledgements}

We thank Chris Paton, Mick Townsend and Gavin Omodei for assistance with mosquito collections; Luke Ambrose for laboratory assistance; Colonel Robert D. Cooper for assistance with analyses of mosquito reproductive status; and Captain Weng Chow and Colonel Cooper for sharing unpublished data.

\section{Funding}

This work was supported by grants (to GED) from the Eck Institute for Global Health, University of Notre Dame (UND), a UND Faculty Research Support Program Initiation Grant, and the NIGMS R01-GM087508; a UND College of Science-Summer Undergraduate Research Fellowship and a Hoffman Fellowship (to GFG); a Balfour-Hesburgh Research Fellowship (to DJA); Eck Institute for Global Health Undergraduate research awards to DJA and GFG; and grants to TRB from the Bill \& Melinda Gates Foundation (grant no. 45114) and the National Institutes of Health for the International Center of Excellence in Malaria Research in the southwest Pacific (Award No. SU19A1089686). SAR was supported by a National Health and Medical Research Council Senior Research Fellowship 1044698.

\section{Availability of data and materials}

Data supporting the conclusions of this article are included within the article and its additional files. The datasets used and/or analyzed during the present study available from the corresponding author upon reasonable request. 


\section{Authors' contributions}

GED conceived the study and its design. GED, SAR and TRB collected mosquitoes from the field, and GED performed the mosquito flight activity experiments. TRB and GED conducted reproductive state analysis, and NWB completed molecular analysis of mosquito samples. ADS, DJA, GFG and GED analyzed the data, DJA and GED wrote the manuscript, and TRB, SAR and TRB contributed revisions. All authors read and approved the final manuscript.

\section{Ethics approval and consent to participate}

Not applicable.

\section{Consent for publication}

Not applicable.

\section{Competing interests}

The authors declare that they have no competing interests.

\section{Publisher's Note}

Springer Nature remains neutral with regard to jurisdictional claims in published maps and institutional affiliations.

\section{Author details}

'Department of Biological Sciences and Eck Institute for Global Health, Galvin Life Science Center, University of Notre Dame, Notre Dame, IN 46556, USA. 2University of Queensland, School of Biology, St Lucia, Queensland, Australia. ${ }^{3}$ CSIRO, Dutton Park, Queensland, Australia. ${ }^{4}$ Australian Institute of Tropical Health and Medicine, James Cook University, Cairns, Queensland, Australia.

Received: 1 June 2018 Accepted: 18 December 2018

Published online: 22 January 2019

\section{References}

1. Beebe NW, Russell T, Burkot TR, Cooper RD. Anopheles punctulatus group: evolution, distribution, and control. Annu Rev Entomol. 2015;60:335-50.

2. Black RH. Malaria in Australia. Canberra: School of Public Health and Tropical Medicine, The University of Sydney Publication No. 9 Australian Government Publishing Services; 1972.

3. Ford E. The malaria problem in Australia and the Australian Pacific territories. Med J Aust. 1950;1:749-60.

4. Burkot TR, Graves PM, Paru R, Lagog M. Mixed blood feeding by the malaria vectors in the Anopheles punctulatus complex (Diptera: Culicidae). J Med Entomol. 1988;25:205-13.

5. Mackerras IM. The Australasian anophelines as vectors of malaria. Med J Aust. 1947;1:1-8.

6. Spencer M. Malaria: The Australian Experience, 1843-1991. Townsville: Australasian College of Tropical Medicine Inc.; 1994.

7. Hanna JN, Ritchie SA, Eisen DP, Cooper RD, Brookes DL, Montgomery BL. An outbreak of Plasmodium vivax malaria in Far North Queensland, 2002. Med J Aust. 2004;180:24-8.

8. Charlwood JD, Paru R, Dagoro H, Lagog M. Influence of moonlight and gonotrophic age on biting activity of Anopheles farauti (Diptera: Culicidae) from Papua New Guinea. J Med Entomol. 1986;23:132-5.

9. Bell D, Cameron A, Fernando M, Pholsyna K, Foley D, Bakote'e B, et al. Malaria in Honiara, Solomon Islands: vector studies. Southeast Asian J Trop Med Public Health. 1996;27:372-7.

10. Benet A, Mai A, Bockarie F, Lagog M, Zimmerman P, Alpers MP, et al. Polymerase chain reaction diagnosis and the changing pattern of vecto ecology and malaria transmission dynamics in Papua New Guinea. Am J Trop Med Hyg. 2004;71:277-84

11. Bugoro H, Iro'ofa C, Mackenzie DO, Apairamo A, Hevalao W, Corcoran S, et al. Changes in vector species composition and current vector biology and behaviour will favour malaria elimination in Santa Isabel Province, Solomon Islands. Malar J. 2011;10:287.

12. Bangs MJ, Taai K, Howard TM, Cook S, Harbach RE. The mosquito Anopheles (Cellia) oreios sp. n., formerly species 6 of the Australasian Anopheles farauti complex, and a critical review of its biology and relation to disease. Med Vet Entomol. 2015;29:68-81.

13. Sweeney AW. Anopheles farauti no. 2 as a possible vector of malaria in Australia. Trans R Soc Trop Med Hyg. 1980;74:830-1.

14. Cooper RD, Waterson DGE, Frances SP, Beebe NW, Pluess B, Sweeney AW. Malaria vectors of Papua New Guinea. Int J Parasitol. 2009;39:1495-501.
15. Sinka ME, Bangs MJ, Manguin S, Chareonviriyaphap T, Patil AP, Temperley WH, et al. The dominant Anopheles vectors of human malaria in the AsiaPacific region: occurrence data, distribution maps and bionomic précis. Parasit Vectors. 2011:4:89.

16. Russell TL, Beebe NW, Bugoro H, Apairamo A, Cooper RD, Collins FH, et al. Determinants of host feeding success by Anopheles farauti. Malar J. 2016;15: 152.

17. Burkot TR, Bugoro H, Apairamo A, Cooper RD, Echeverry DF, Odabasi D, et al. Spatial-temporal heterogeneity in malaria receptivity is best estimated by vector biting rates in areas nearing elimination. Parasit Vectors. 2018;11:606

18. Russell TL, Beebe NW, Cooper RD, Lobo NF, Burkot TR. Successful malaria elimination strategies require interventions that target changing vector behaviours. Malar J. 2013:12:56

19. Ambrose L, Cooper RD, Russell TL, Burkot TR, Lobo NF, Collins FH, et al. Microsatellite and mitochondrial markers reveal strong gene flow barriers for Anopheles farauti in the Solomon Archipelago: implications for malaria vector control. Int J Parasitol. 2014:44:225-33.

20. Taylor B. Changes in the feeding behaviour of a malaria vector, Anopheles farauti Lav., following use of DDT as a residual spray in houses in the British Solomon Islands Protectorate. Trans R Entomol Soc London. 1975;127:277-92.

21. Burkot TR, Russell TL, Reimer $L$, Bugoro H, Beebe NW, Cooper RD, et al. Barrier screens: a method to sample blood-fed and host-seeking exophilic mosquitoes. Malar J. 2013;12:49.

22. Russell TL, Beebe NW, Bugoro H, Apairamo A, Collins FH, Cooper RD, et al. Anopheles farauti is a homogeneous population that blood feeds early and outdoors in the Solomon Islands. Malar J. 2016;15:151.

23. Charlwood JD, Graves PM. The effect of permethrin-impregnated bednets on a population of Anopheles farauti in coastal Papua New Guinea. Med Vet Entomol. 1987;1:319-27.

24. Moiroux N, Gomez MB, Pennetier C, Elanga E, Djènontin A, Chandre F, et al. Changes in Anopheles funestus biting behavior following universal coverage of long-lasting insecticidal nets in Benin. J Infect Dis. 2012;206:1622-9.

25. Takken W. Do insecticide-treated bednets have an effect on malaria vectors? Trop Med Int Heal. 2002;7:1022-30.

26. Sougoufara S, Diédhiou SM, Doucouré S, Diagne N, Sembène PM, Harry M, et al. Biting by Anopheles funestus in broad daylight after use of long-lasting insecticidal nets: a new challenge to malaria elimination. Malar J. 2014;13:125.

27. Thomsen EK, Koimbu G, Pulford J, Jamea-Maiasa S, Ura Y, Keven JB, et al. Mosquito behavior change after distribution of bednets results in decreased protection against malaria exposure. J Infect Dis. 2017;215:790-7.

28. Pates $\mathrm{H}$, Curtis C. Mosquito behavior and vector control. Annu Rev Entomol. 2005;50:53-70

29. Ranson H, N'guessan R, Lines J, Moiroux N, Nkuni Z, Corbel V. Pyrethroid resistance in African anopheline mosquitoes: what are the implications for malaria control? Trends Parasitol. 2011:27:91-8.

30. Sheppard AD, Rund SSC, George GF, Clark E, Acri DJ, Duffield GE. Light manipulation of mosquito behaviour: acute and sustained photic suppression of biting activity in the Anopheles gambiae malaria mosquito. Parasit Vectors. 2017:10:255.

31. Das S, Dimopoulos G. Molecular analysis of photic inhibition of bloodfeeding in Anopheles gambiae. BMC Physiol. 2008;8:23.

32. Jones MDR, Cubbin CM, Marsh D. The circadian rhythm of flight activity of the mosquito Anopheles gambiae: the light-response rhythm. J Exp Biol. 1972;57:337-46

33. Leming MT, Rund SSC, Behura SK, Duffield GE, O'Tousa JE. A database of circadian and diel rhythmic gene expression in the yellow fever mosquito Aedes aegypti. BMC Genomics. 2014;15:1128.

34. Rund SSC, Hou TY, Ward SM, Collins FH, Duffield GE. Genome-wide profiling of diel and circadian gene expression in the malaria vector Anopheles gambiae. Proc Natl Acad Sci USA. 2011;108:E421-30.

35. Rund SSC, Lee SJ, Bush BR, Duffield GE. Strain- and sex-specific differences in daily flight activity and the circadian clock of Anopheles gambiae mosquitoes. J Insect Physiol. 2012;58:1609-19.

36. Rund SS, Gentile JE, Duffield GE. Extensive circadian and light regulation of the transcriptome in the malaria mosquito Anopheles gambiae. BMC Genomics. 2013;14:218.

37. Clements AN. The Biology of Mosquitoes, Vol. II Sensory Reception and Behaviour. Wallingford: CABI Publishing; 1999

38. Rund SSC, Bonar NA, Champion MM, Ghazi JP, Houk CM, Leming MT, et al. Daily rhythms in antennal protein and olfactory sensitivity in the malaria mosquito Anopheles gambiae. Sci Rep. 2013;3:2494. 
39. Haddow AJ, Ssenkubuge Y. Laboratory observations on the ovipositioncycle in the mosquito Anopheles (Cellia) gambiae Giles. Ann Trop Med Parasitol. 1962;56:352-5.

40. Jones MDR, Gubbins SJ. Changes in the circadian flight activity of the mosquito Anopheles gambiae in relation to insemination, feeding and oviposition. Physiol Entomol. 1978;3:213-20.

41. Balmert NJ, Rund SSC, Ghazi JP, Zhou P, Duffield GE. Time-of-day specific changes in metabolic detoxification and insecticide resistance in the malaria mosquito Anopheles gambiae. J Insect Physiol. 2014;64:30-9.

42. Dunlap JC, Loros JJ, Decoursey PJ. Chronobiology: Biological Timekeeping. Sunderland, Massachusetts: Sinauer Associates, Inc.; 2004

43. Afifi SE, Spencer M, Hudson PB, Tavil NW. Biting prevalence and malaria transmission patterns in the Anopheles punctulatus complex (Diptera: Culicidae) in Papua New Guinea. Aust J Exp Biol Med Sci. 1980;58:1-17.

44. Bugoro H, Cooper RD, Butafa C, Iro'ofa C, Mackenzie DO, Chen C, et al. Bionomics of the malaria vector Anopheles farauti in Temotu Province, Solomon Islands: issues for malaria elimination. Malar J. 2011:10:133.

45. Bugoro H, Hii JL, Butafa C, Iro'ofa C, Apairamo A, Cooper RD, et al. The bionomics of the malaria vector Anopheles farauti in Northern Guadalcanal Solomon Islands: issues for successful vector control. Malar J. 2014;13:56.

46. Taylor B. Control of flight activity in mosquitoes. Nature. 1977:265:762.

47. Taylor B. Geographical range and circadian rhythms. Nature. 1969;222:296-7.

48. Jones MDR. The programming of circadian flight-activity in relation to mating and the gonotrophic cycle in the mosquito, Aedes aegypti. Physiol Entomol. 1981;6:307-13.

49. Rowland M. Changes in the circadian flight activity of the mosquito Anopheles stephensi associated with insemination, blood-feeding, oviposition and nocturnal light intensity. Physiol Entomol. 1989;14:77-84.

50. Ritchie SA, Cortis G, Paton C, Townsend M, Shroyer D, Zborowski P, et al. A simple non-powered passive trap for the collection of mosquitoes for arbovirus surveillance. J Med Entomol. 2013;50:185-94.

51. Detinova TS. Age-grouping methods in Diptera of medical importance with special reference to some vectors of malaria. Monogr Ser Wld HIth Organ. 1962:47:13-191.

52. Hugo LE, Quick-Miles S, Kay BH, Ryan PA. Evaluations of mosquito age grading techniques based on morphological changes. J Med Entomol. 2008; 45:353-69.

53. Beebe NW, Ellis JT, Cooper RD, Saul A. DNA sequence analysis of the ribosomal DNA ITS2 region for the Anopheles punctulatus group of mosquitoes. Insect Mol Biol. 1999:8:381-90.

54. Beebe NW, Saul A. Discrimination of all members of the Anopheles punctulatus complex by polymerase chain reaction-restriction fragment length polymorphism analysis. Am J Trop Med Hyg. 1995;53:478-81.

55. Jones MDR, Hill M, Hope AM. The circadian flight activity of the mosquito Anopheles gambiae: phase setting by the light regime. J Exp Biol. 1967;47: 503-11.

56. Gary RE, Foster WA. Diel timing and frequency of sugar feeding in the mosquito Anopheles gambiae, depending on sex, gonotrophic state and resource availability. Med Vet Entomol. 2006;20:308-16.

57. Clements AN, Kerkut GA. The Physiology of Mosquitoes: International Series of Monographs. Oxford: Pergamon Press Ltd.; 1963.

58. Gillies MT. Studies in house leaving and outside resting of Anopheles gambiae Giles and Anopheles funestus Giles in East Africa: II. The exodus from houses and the house resting population. Bull Entomol Res. 1954;45:375-87.

59. Gillies MT. Studies of house leaving and outside resting of Anopheles gambiae Giles and Anopheles funestus Giles in east Africa. I. The outside resting population. Bull Entomol Res. 1954;45:361-73.

60. Charlwood JD, Jones MDR. Mating in the mosquito, Anopheles gambiae s.l. II. Swarming behaviour. Physiol Entomol. 1980;5:315-20.

61. Charlwood JD, Pinto J, Sousa CA, Madsen H, Ferreira C, do Rosario VE. The swarming and mating behaviour of Anopheles gambiae s.s. (Diptera: Culicidae) from São Tomé Island. J Vector Ecol. 2002;27:178-83.

62. Manoukis NC, Diabate A, Abdoulaye A, Diallo M, Dao A, Yaro AS, et al. Structure and dynamics of male swarms of Anopheles gambiae. J Med Entomol. 2009:46:227-35.

63. Sumba LA, Okoth K, Deng AL, Githure J, Knols BGJ, Beier JC, et al. Daily oviposition patterns of the African malaria mosquito Anopheles gambiae Giles (Diptera: Culicidae) on different types of aqueous substrates. J Circadian Rhythms. 2004;2:6.

64. Daggy RH. The biology and seasonal cycle of Anopheles farauti on Espiritu Santo, New Hebrides. Ann Entomol Soc Am. 1945;38:3-13.
65. Bockarie MJ, Alexander N, Bockarie F, Ibam E, Barnish G, Alpers M. The late biting habit of parous Anopheles mosquitoes and pre-bedtime exposure of humans to infective female mosquitoes. Trans R Soc Trop Med Hyg. 1996; 90:23-5.

66. Charlwood JD, Wilkes TJ. Studies on the age-composition of samples of Anopheles darlingi Root (Diptera: Culicidae) in Brazil. Bull Entomol Res. 1979; 69:337-42.

67. Charlwood JD, Graves PM, Marshall TF. Evidence for a 'memorized' home range in Anopheles farauti females from Papua New Guinea. Med Vet Entomol. 1988;2:101-8.

68. Molineaux L, Gramiccia G. The Garki Project. Research on the epidemiology and control of malaria in the Sudan Savanna of West Africa. Geneva: World Health Organization; 1980

69. Howell PI, Knols BGJ. Male mating biology. Malar J. 2009:8:S8.

70. Sawadogo S, Costantini C, Pennetier C, Diabaté A, Gibson G, Dabiré R. Differences in timing of mating swarms in sympatric populations of Anopheles coluzzii and Anopheles gambiae s.s. (formerly An. gambiae M and S molecular forms) in Burkina Faso, West Africa. Parasit Vectors. 2013;6:275.

71. Jones MDR, Gubbins SJ, Cubbin CM. Circadian flight activity in four sibling species of the Anopheles gambiae complex (Diptera, Culicidae). Bull Entomol Res. 1974;64:241-6.
Ready to submit your research? Choose BMC and benefit from:

- fast, convenient online submission

- thorough peer review by experienced researchers in your field

- rapid publication on acceptance

- support for research data, including large and complex data types

- gold Open Access which fosters wider collaboration and increased citations

- maximum visibility for your research: over $100 \mathrm{M}$ website views per year

At $\mathrm{BMC}$, research is always in progress.

Learn more biomedcentral.com/submissions 